Revista Mexicana de Economía y Finanzas Nueva Época

Volumen 14 Número 4, Octubre - Diciembre 2019, pp. 693-714

DOI: https://doi.org/10.21919/remef.v14i4.352

\title{
Co-movimientos entre los Índices Accionarios y los Ciclos Económicos de Estados Unidos y México
}

\author{
Luis Ignacio Román de la Sancha ${ }^{1}$ \\ Universidad Nacional Autónoma de México, México \\ Federico Hernández Álvarez \\ Universidad Nacional Autónoma de México, México \\ Gabriel Rodríguez García \\ Consultoría B\&G Business \& Growth S.C., México \\ Universidad Nacional Autónoma de México, México \\ (Recepción: 13/noviembre/2018, aceptado: 15/abril/2019)
}

\section{Resumen}

El objetivo de este trabajo es identificar los co-movimientos entre los índices accionarios Dow Jones (DJ) de los Estados Unidos (EU) y el Índice de Precios y Cotizaciones (IPC) de México; así también con los ciclos económicos de ambos países. Para explicar esta relación se propone una metodología que comprende conceptos y modelos utilizados en el estudio de ciclos económicos, en particular: tendencia, ciclo, puntos de giro, conformidad y sincronía. Los resultados muestran que las tendencias del índice DJ y el indicador de la economía mexicana (SICCA) muestran una alta conformidad. Por otra parte, los ciclos financieros del DJ e IPC presentan una sincronía casi coincidente. Algunas variables que escapan a este trabajo y que también influyen en los ciclos económicos incluyen la tasa de cambio, ciclos crediticios y políticos. La originalidad de esta investigación consiste en el estudio de la relación que tienen los ciclos financieros obtenidos de los principales índices accionarios con los ciclos económicos. Se concluye que los ciclos del DJ e IPC anticipan con al menos cuatro meses a los ciclos económicos de EU y México.

Clasificación JEL: E32, G10, C10, C63

Palabras clave: Ciclos Económicos, Ciclos Financieros, Co-movimientos, Filtro Hodrick y Prescott, Algoritmo Bry y Boschan

\section{Co-movements between Stock Market Indices and Economic cycles: The case of US and Mexico}

\section{Abstract}

The aim of this paper is to identify the co-movements between the Dow Jones stock index (DJ) of the United States of America (US) and the Índice de Precios y Cotizaciones (IPC) of Mexico along with the economic cycles of both countries. A novel approach using concepts and models widely accepted in the study of economic cycles is developed to explain this relationship, in particular: trend, cycle, turning points, conformity and synchrony. Finding points show that trend of the DJ index and the mexican economy indicator (SICCA) present high conformity. On the other hand, the financial cycles of the DJ and IPC present an almost coincident synchrony. Some variables such as exchange rate, credit and political cycles that also influence economic cycles are not taken into account. The originality of this paper is the study of the relationship between financial cycles obtained from the main stock indexes and economic cycles. It is concluded that the cycles of the DJ and IPC anticipate economic cycles of both US and Mexico with at least four months.

\footnotetext{
${ }^{1}$ Av. Universidad No 3000, Universidad Nacional Autónoma de México, C.U., Ciudad de México, C.P. 04510, celular: 044 55-27-27-37-08, e-mail: roman.luisignacio@gmail.com
} 


\section{Abstract}

JEL Classification: E32, G10, C10, C63

Keywords: Economic Cycles, Financial Cycles, Co-movements, Hodrick 86 Prescott Filter, Bry $\&$ Boschan algorithm

\section{Introducción}

Los ciclos financieros han recibido mayor atención a partir de las crisis económicas originadas en los mercados financieros y su posterior efecto en la economía real (Grinderslev et al., 2017). Por otra parte, el desarrollo de los estudios sobre ciclos económicos es amplio, tanto en México como en otros países. Este trabajo pretende estudiar los co-movimientos o relaciones que tienen los ciclos financieros obtenidos a través de índices accionarios de los mercados financieros y los ciclos económicos de Estados Unidos y México para el periodo noviembre de 1991 a abril de 2018. En particular, se utilizan los conceptos de tendencia, ciclo, puntos de giro y su fechado, conformidad y sincronía, ampliamente reconocidos en el estudio de ciclos económicos (Claessens et al., 2011).

De acuerdo con Heath (2011), a partir del 2000, el INEGI (Instituto Nacional de Estadística y Geografía) inició a publicar información necesaria para conocer los ciclos económicos en México. Sin embargo, aún no existen estudios acerca de ciclos financieros en México, y en otros países la literatura es escasa sobre este tema.

En este trabajo se presentan indicadores (i.e. tendencia, ciclo, puntos de giro y su fechado, conformidad y sincronía) que pretenden explicar la relación de los ciclos económicos de Estados Unidos y México con los ciclos accionarios de los principales mercados financieros de estos países, que aún no es deducida. La metodología propuesta busca identificar los co-movimientos de los índices representativos del mercado financiero mexicano y estadounidense con los ciclos de sus respectivas economías, así como destacar la relación que estos ciclos tienen con las principales crisis financieras del periodo en estudio.

Los estudios acerca de ciclos económicos y crisis en mercados financieros son vastos, sin embargo, ninguno de éstos describe la relación entre los ciclos económicos y los ciclos financieros obtenidos a partir de los mercados financieros. Por ejemplo Cuevas et al. (2003) estudia el grado de sincronización macroeconómica de México y sus socios comerciales del TLCAN (Tratado de Libre Comercio de América del Norte) utilizando una metodología que toma en cuenta diversos sectores de la actividad económica, componentes de la oferta y demanda agregadas de cada país y la evolución regional del empleo tanto en México como en Estados Unidos, concluyendo que a partir de la entrada en vigor del TLCAN (1994) la sincronización macroeconómica ha aumentado considerablemente, pero que las perturbaciones idiosincráticas continúan ejerciendo un efecto importante en el ciclo económico de México. En el mismo sentido, Calderón Villareal y Hernández Bielma (2017) analizan la peculiaridad de la dinámica de las fluctuaciones económicas de la economía mexicana en el marco de su integración con Estados Unidos y Canadá. Para ello seleccionan variables macroeconómicas (i.e., exportaciones, importaciones, gasto público, inversión privada, inversión pública) que expliquen la relación del ciclo económico en México para el periodo 1993-2013 con el ciclo económico de Estados Unidos, tomando en cuenta el grado de sincronización de los ciclos y los co-movimientos de dichas variables. Sus resultados demuestran cómo la economía mexicana homogeneizó las crisis provenientes de Estados Unidos (2001 y 2007) y cómo los ciclos económicos de ambos países se fueron alineando a partir de la entrada en vigor del TLCAN (1994). Asimismo, Heath (2011) realiza un amplio estudio de la identificación de los ciclos económicos en México de los últimos 30 años, encontrando que de 1980 a 2009 se han tenido seis recesiones, que coinciden con el número de sexenios. En este trabajo Heath destaca la publicación 
del Sistema de Indicadores Compuestos Coincidente y Adelantado (SICCA) por parte del INEGI, mencionando que es una mapeo fiel de los ciclos económicos de México a partir de 1980. De igual forma, Antón (2011) estudia las propiedades del ciclo económico en México y su relación con los ciclos de las economías desarrolladas de la OCDE (Organización para la Cooperación y el Desarrollo Económicos). En este trabajo Antón menciona que la duración de las expansiones en México son menores, en comparación las de las economías de la OCDE; por el contrario de las recesiones, las cuales tienen una duración mayor en México que en el promedio de estas economías. De igual forma Antón (2011) observó que previó al año 1996 la correlación de los ciclos del PIB de México y Estados Unidos era débil; fue después de ese año, es decir, poco después de la entrada en vigor del TLCAN (1994), que ambos componentes cíclicos se comportan de manera muy similar. Por su parte, Mejía-Reyes y Campos-Chávez (2009) estudian el grado de sincronización de los ciclos económicos de 17 estados mexicanos con el ciclo económico de Estados Unidos, con el fin de observar la dinámica de ciclos de negocios específicos de dichos estados con el de la economía estadounidense. Para estudiar dicha relación hicieron uso del Filtro Hodrick y Prescott (HP) (1997) para medir los co-movimientos de los ciclos de negocio para el periodo 1993-2007. Sus resultados muestran que existe una alta sincronización del ciclo compuesto económico de México (de los 17 estados mexicanos) con el ciclo económico de Estados Unidos.

En cuanto a la relación de la economía de Estados Unidos con el mercado financiero mexicano, Jaramillo Olivares y Jaramillo Jaramillo (2016) analizaron los efectos de la crisis del 2008 en las empresas listadas en la Bolsa Mexicana de Valores (BMV). Los resultados indicaron que el sector industrial y principalmente el de la construcción fueron los más afectados, lo que posteriormente tuvo efectos negativos en la economía mexicana.

En relación con los ciclos financieros, (Claessens et al., 2011) realiza un estudio de los ciclos crediticios, precios de vivienda y precios de las acciones para 21 países desarrollados que forman parte de la OCDE, utilizando la tradición de Burns y Mitchel (1946) para determinar puntos de giro y su fechado de ciclos de negocio. Sus resultados muestran que los ciclos financieros obtenidos de los mercados financieros (precio de las acciones) y el ciclo de vivienda (precio de vivienda) son más largos y pronunciados que los ciclos crediticios para los 21 países de estudio. En el mismo sentido, Karfakis y Karfaki (2018) estudian si los ciclos financieros obtenidos a través de crédito otorgado a diversos sectores productivos, tasas de interés a tres meses, y el índice del consumidor, son indicadores del comportamiento de la economía, medida a través del producto interno bruto (PIB), durante expansiones y recesiones en Grecia, concluyendo que dichos ciclos son un indicador importante de la economía griega.

Con respecto a las crisis financieras Claessens y Kose (2013) estudian sus características, los tipos de crisis que se dan con mayor frecuencia y sus efectos en la economía real. El estudio revela que existen cuatro tipos de crisis financieras: crisis monetaria, crisis sudden stop (parada repentina), crisis de deuda y crisis bancaria. Asimismo, el análisis histórico que realizan muestra como pueden coexistir los cuatro tipos de crisis en un evento. En este estudio también mencionan que estas crisis vienen una tras otra y se ha intensificado la frecuencia con que suceden debido a la liberalización de los mercados financieros y la cada vez mayor integración económica-financiera del mundo. Por su parte, Sosa et al. (2017) estudió el impacto de la crisis financiera subprime del 2007 en la dinámica de los mercados accionarios más importantes de los continentes americano y europeo a partir de modelos GARCH simétricos y asimétricos para el periodo 2003-2015, encontrando que dicha crisis impacto en el comportamiento de las bolsas de valores, incrementando su volatilidad y asimetria. En el mismo sentido, Cruz-Aké et al. (2012) realiza un estudio donde muestran evidencia empírica sobre la sincronización de los índices bursátiles de las principales economías del mundo, la cual aumenta cuando se presentan periodos de alta volatilidad derivado de crisis en los mercados financieros. Asimismo, Rocha Armada et al. 
(2011) estudió los efectos de contagio de las crisis financieras en los mercados bursátiles de nueve países desarrollados a través de pruebas empíricas: pruebas de correlación; de Kolmogorov-Smirnov, entre otras. Sus resultados muestra que existe evidencia suficiente para demostrar efectos de contagio durante la crisis asiática y rusa de 1997 y la crisis de Estados Unidos del 2001. En el caso de México, Flores Paredes y Madrueño Aguilar (2006) analizan la relación del ciclo económico mexicano con las crisis financieras de 1970 a 2006. Para explicar esta relación toman en cuenta la dinámica de variables macroeconómicas (i.e. PIB per cápita, crecimiento en salarios reales e inversión extranjera directa). Sus resultados muestran que las crisis que enfrentaron los gobiernos de Luis Echeverría (1970-1976) y López Portillo (1976-1982) fueron producto de una insostenible política fiscal y una política cambiaria fija o semifija. Asimismo, explican que la crisis de 1994 fue producto de contradicciones en la política fiscal y cambiaria en un entorno de globalización y liberalización financiera, además de incurrir en un riesgo moral por parte del sector bancario. Finalmente, mencionan que para explicar las crisis recientes del siglo XXI se han propuesto modelos como el de contagio bursátil, correlaciones en el movimiento de indicadores financieros-económicos, fragilidad financiera y riesgo moral. Con dichos modelos explicaron el estancamiento económico del 2000 al 2003 originada por la crisis dotcom, encontrando que la sincronía en los ciclos económicos de México y Estados Unidos es alta. Particularmente, la crisis mexicana de 1994 es estudiada por Bezis y Petit (1997), mostrando que los datos estadísticos disponibles en ese momento habrían sido suficientes para anticipar el colapso de la economía mexicana, haciendo uso de puntos de giro y su fechado como indicadores económico-financieros. Asimismo, destacan que las series financieras son un buen indicador para anticipar los puntos de giro en la economía de un país.

Por la anterior discusión de la literatura sobre ciclos económicos, ciclos financieros y crisis financieras, se muestra que no hay estudios que investiguen sobre la relación de los ciclos financieros obtenidos a través de índices accionarios con los ciclos económicos de Estados Unidos y México. Asimismo, no se realiza un análisis en términos de co-movimientos en tendencia y ciclos de las crisis financieras que surgen en mercados financieros y como impactan en la economía de un país.

Las hipótesis principales de este trabajo son que la tendencia, comportamiento de largo plazo, del índice DJ y el indicador coincidente del SICCA se expanden y contraen en los mismos periodos de tiempo, presentando así una alta conformidad. Por otra parte, la tendencia del SICCA y del IPC en ocasiones presentan momentos de baja y alta conformidad, es decir, en ocasiones la tendencia accionaria mexicana no coincide con la dinámica económica nacional. Respecto al análisis de ciclos, se tiene la hipótesis de que los ciclos financieros de los índices DJ e IPC anticipan con al menos cuatro meses a los ciclos económicos de EU y México.

La aportación principal de esta investigación respecto a la literatura existente sobre ciclos económicos en México, el estudio de los ciclos financieros en otros países y las crisis financieras, es explicar la relación que existe entre los índices accionarios de los principales mercados financieros de México y Estados Unidos con los ciclos económicos de ambos países, haciendo un análisis de las crisis financieras para el periodo noviembre de 1991 a abril de 2018. De igual forma, se contribuye con una metodología que; en primer lugar haciendo uso del Filtro Hodrick y Prescott (HP) (1997) permite obtener la tendencia y el ciclo de una serie de tiempo; segundo, se aplica el algoritmo Bry y Boschan (B\&B) (1971), el cual está formulado conforme a la experiencia del NBER (National Bureau of Economic Research), para la determinación de puntos de giro y su fechado; por último, se utilizan los conceptos de conformidad y sincronía para explicar los co-movimientos de las series financieras y económicas, de acuerdo con Moore \& Shiskin (1967). Lo anterior revela una explicación que aún no es deducida en términos económico-financieros sobre la relación México-Estados Unidos, así como su relación con las crisis financieras más 
relevantes del periodo en estudio.

El trabajo se divide en cinco secciones: en la primera, introducción, se realiza una discusión del estado del arte de los ciclos económicos, ciclos financieros y crisis financieras; en la segunda se detallan los datos que se utilizaron, en particular los índices y fechados con los que se representa la economía mexicana y estadounidense. En la tercera sección se explica la propuesta metodológica, la cual incluye la aplicación de los métodos y algoritmos antes mencionados. En la cuarta sección, se discuten los principales resultados que se obtuvieron. En la última sección, se presentan las conclusiones y aportaciones.

\section{Datos}

Los datos utilizados para este estudio abarcan el periodo de noviembre de 1991 a abril de 2018; para los ciclos financieros se emplearon dos indicadores de mercado, Dow Jones e IPC para Estados Unidos y México, respectivamente, los cuales son considerados como los más representativos del mercado financiero en ambos países. Para los ciclos económicos, se utilizó el índice compuesto coincidente del SICCA para México, y el fechado del NBER para Estados Unidos. Así también, se muestran las fechas de las crisis financieras más relevantes durante el periodo de estudio.

\subsection{Dow Jones}

El índice bursátil Dow Jones, mide el desempeño de las 30 mayores empresas que cotizan en el mercado bursátil de Estados Unidos. En la Figura 1, se muestra la serie del DJ de noviembre de 1991 a abril de 2018, así también las fechas de inicio y finalización de los valles y picos de sus ciclos observables, determinados con el algoritmo de B\&B. En particular, para este periodo, el DJ presenta tres periodos de recesión, asociados a las crisis: Burbuja Tecnológica (2001), Hipotecas subprime (2007) y Crisis del mercado financiero chino (2015), ver Tabla 3.

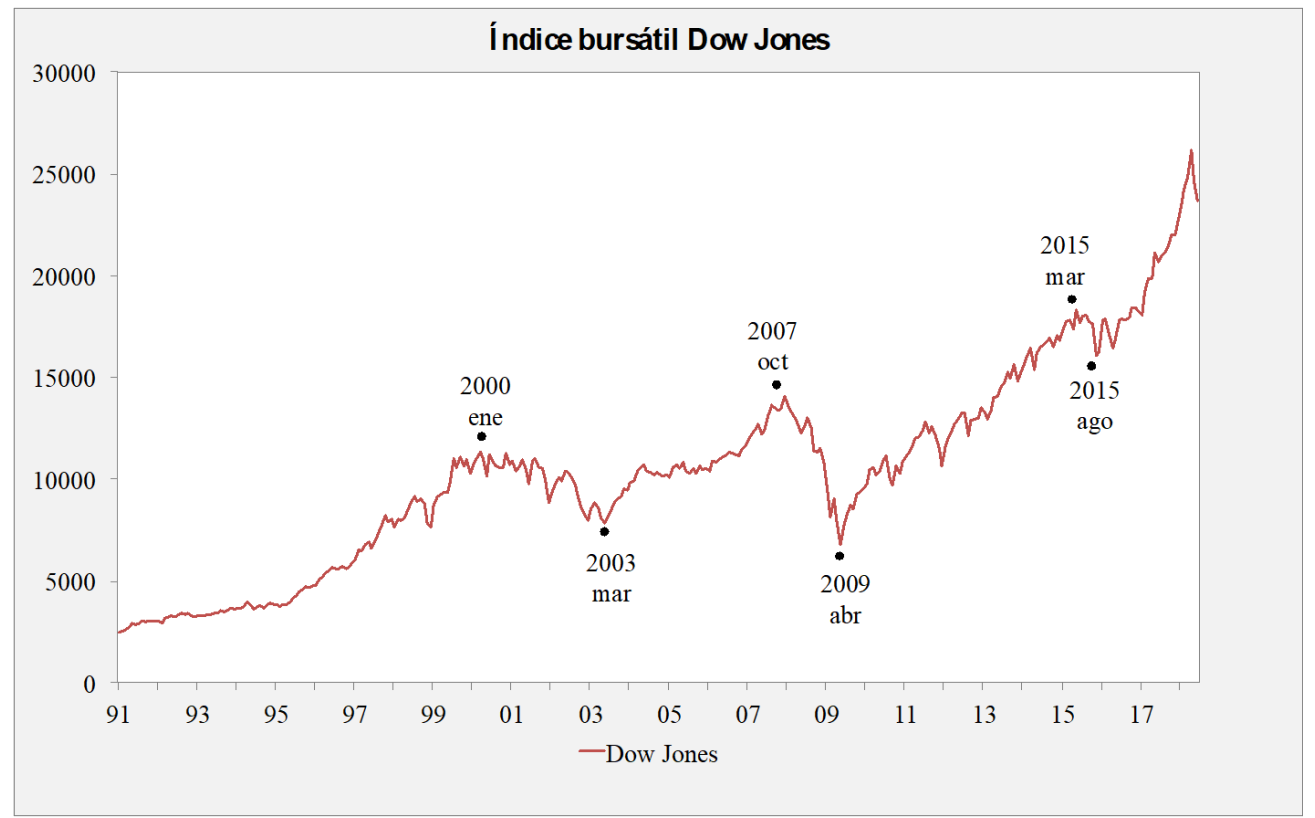

Figura 1. Índice Dow Jones (DJ) de noviembre de 1991 a abril de 2018, con las fechas de sus puntos de giro determinadas con el algoritmo de B\&B. Fuente: elaboración propia con información de Yahoo Finance. 


\subsection{IPC}

El Índice de Precios y Cotizaciones (IPC) es el principal índice bursátil en México, su muestra concentra las 35 principales empresas listadas en la Bolsa Mexicana de Valores (BMV). En la Figura 2, se grafica la serie del IPC de noviembre de 1991 a abril de 2018, así también, las fechas de los picos y valles de sus ciclos, encontrados por el algoritmo de B\&B. En particular, este índice fue afectado por las ocho crisis financieras ocurridas durante este periodo, ver Tabla 3.

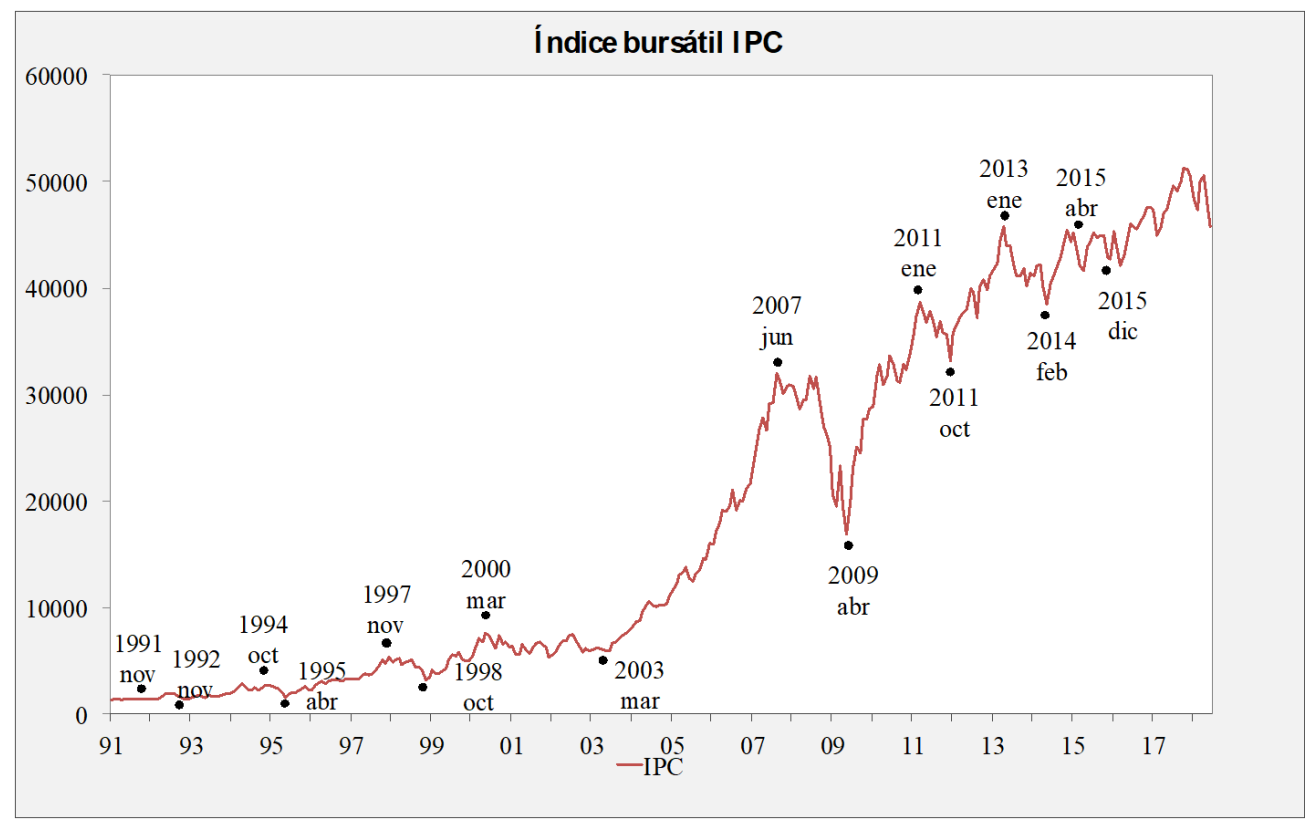

Figura 2. Índice de Precios y Cotizaciones (IPC) de noviembre de 1991 a abril de 2018, con las fechas de sus puntos de giro determinadas con el algoritmo de B\&B.

Fuente: elaboración propia con información de Yahoo Finance.

\subsection{SICCA}

El Sistema de Indicadores Compuestos: Coincidente y Adelantado (SICCA) de acuerdo con la definición del INEGI (2010) es un indicador que permite conocer oportunamente la actividad económica en México. Este sistema incorpora información de diversos mercados del país que permiten anticipar la posible trayectoria de la economía nacional (INEGI, 2010). En este trabajo se utiliza el indicador coincidente del SICCA.

De acuerdo con el INEGI, el indicador coincidente del SICCA se define de la siguiente manera:

El indicador coincidente es un índice que incluye series económicas con información sobre el mercado de bienes y servicios y sobre el mercado laboral. En particular dicho indicador incorpora el Producto Interno Bruto Mensual, al Índice de Volumen Físico de la Actividad Industrial, el Número de Asegurados Permanentes del Instituto Mexicano del Seguro Social (IMSS), el Índice de Ventas al por menor en Establecimientos Comerciales y la Tasa de Ocupación Parcial y Desocupación. (INEGI, 2010, p.4).

En la Figura 3, se muestra la serie del componente coincidente del SICCA de noviembre de 1991 a abril de 2018. Para esta serie se observa que fue afectada por las siguientes crisis: Crisis Japonesa (1991), Crisis Mexicana de (1994), Burbuja Tecnológica (2001) e Hipotecas subprime (2007), ver Tabla 3. 


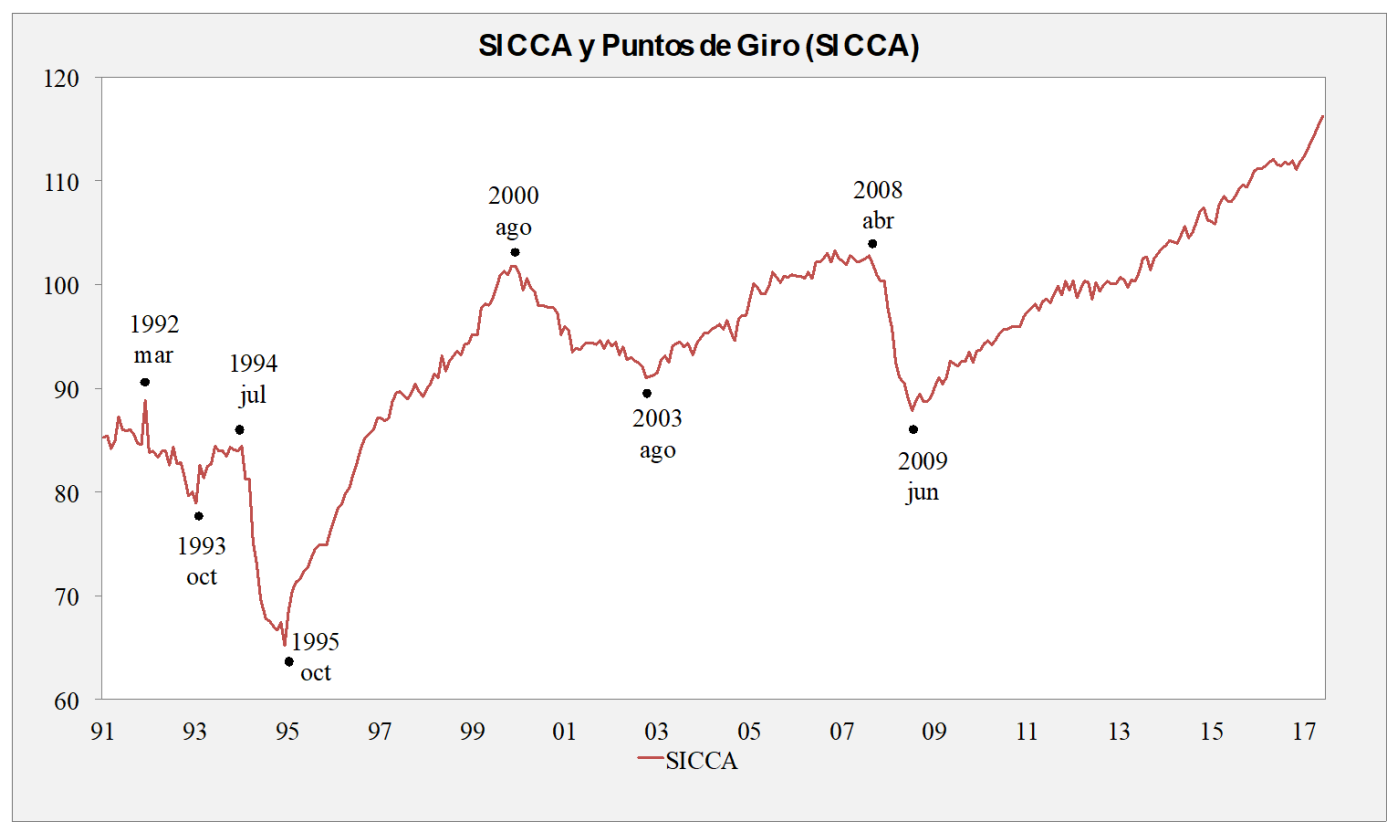

Figura 3. Índice Coincidente del SICCA de noviembre de 1991 a abril de 2018, con las fechas de sus puntos de giro determinadas con el algoritmo de B\&B.

Fuente: elaboración propia con información INEGI.

Asimismo, se señalan las fechas de los picos y valles, asociados a las fases de ciclos económicos, determinados con el algoritmo B\&B, ver Tabla 1.

Tabla 1. Fechado de picos, valles y sus duraciones de los ciclos económicos de México (SICCA).

\begin{tabular}{|c|c|c|c|c|c|}
\hline \multirow{2}{*}{\multicolumn{2}{|c|}{ Fechado }} & \multicolumn{4}{|c|}{ Duración en meses } \\
\hline & & \multirow{2}{*}{$\begin{array}{c}\text { Recesión } \\
\text { Duración, } \\
\text { pico a valle }\end{array}$} & \multirow{2}{*}{$\begin{array}{c}\text { Expansión } \\
\text { valle anterior a } \\
\text { este pico }\end{array}$} & \multicolumn{2}{|c|}{ Ciclo } \\
\hline Pico & Valle & & & $\begin{array}{c}\text { valle a valle } \\
\text { anterior }\end{array}$ & $\begin{array}{c}\text { pico a pico } \\
\text { anterior }\end{array}$ \\
\hline mar-92 & oct-93 & 19 & - & - & - \\
\hline jul-94 & oct-95 & 15 & 9 & 24 & 28 \\
\hline ago-00 & ago-03 & 36 & 58 & 94 & 73 \\
\hline abr-08 & jun-09 & 14 & 56 & 70 & 92 \\
\hline
\end{tabular}

Fuente: Elaboración propia con información de INEGI.

\subsection{Fechado del NBER}

El National Bureau of Economic Research (NBER) de los Estados Unidos se dedica a promover un mejor funcionamiento de la economía (Heath, 2011). Lo anterior, fechando el inicio y fin de recesiones y expansiones de la actividad económica, lo cual se realiza a través de un Comité de Fechas de Ciclos Económicos creado en 1978 y que se ha reconocido como el árbitro oficial de ciclos económicos. En el mismo sentido, en los años 30 y los 40 del siglo XX en el NBER, los ciclos económicos fueron estudiados por A. F. Burns y W. C. Mitchell (1946), quienes implementaron un programa de trabajo para documentar empíricamente los ciclos observados en Estados Unidos y en otros países (Guerrero, 2011).

En la Tabla 2, se presenta las fechas de inicio y terminación de las fases de expansiones y recesiones en Estados Unidos de julio de 1990 a junio de 2009 definidas por el NBER. 
Tabla 2. Fechado de picos, valles y sus duraciones de los ciclos económicos de Estados

\begin{tabular}{|c|c|c|c|c|c|}
\hline \multicolumn{2}{|c|}{ Fechado } & Recesión & $\begin{array}{c}\text { Duración en meses } \\
\text { Expansión }\end{array}$ & \multicolumn{2}{c|}{ Ciclo } \\
\hline Pico & Valle & $\begin{array}{c}\text { Duración, } \\
\text { pico a valle }\end{array}$ & $\begin{array}{c}\text { valle anterior a } \\
\text { este pico }\end{array}$ & $\begin{array}{c}\text { valle a valle } \\
\text { anterior }\end{array}$ & $\begin{array}{c}\text { pico a pico } \\
\text { anterior }\end{array}$ \\
\hline jul- 90 & mar-91 & 8 & 92 & 100 & 108 \\
\hline mar-01 & nov-01 & 8 & 120 & 128 & 128 \\
\hline dic-07 & jun-09 & 18 & 73 & 91 & 81 \\
\hline
\end{tabular}

\subsection{Crisis financieras}

Otro dato importante para el estudio de las relaciones entre los índices accionarios y los ciclos económicos para Estados Unidos y México, es la información acerca de las crisis financieras globales que han acontecido durante los últimos 27 años.

De acuerdo con Claessens y Kose (2013), las crisis financieras tienen elementos comunes, sin embargo, se presentan en diversas formas. Las crisis financieras son asociadas con uno o más fenómenos: cambios sustanciales en el volumen de créditos y los precios de los activos, rupturas severas en la intermediación financiera y el flujo de financiamiento externo a diversos actores de la economía. De igual formal, estos autores identifican cuatro tipos de crisis financieras: crisis monetaria, crisis sudden stop (parada repentina), crisis de deuda y crisis bancaria; mencionan también que estas crisis vienen una tras otra y se ha intensificado la frecuencia con que suceden debido a la liberalización de los mercados financieros y la cada vez mayor integración económica-financiera del mundo. Por ejemplo, Samuelson y Nordhaus (2002) mencionan que las crisis financieras pueden ser ocasionadas por la especulación de los precios de la propia población, si suben los precios en un determinado momento la gente piensa que seguirán subiendo en un futuro, sin tener bases que sustenten el incremento de los precios. Por otra parte, de acuerdo con Wong (2016), los ciclos financieros obtenidos a partir de los mercados financieros son el indicador dominante para detectar un ciclo económico, sin embargo, menciona que no es el único, es importante complementarlo con el análisis de ciclos crediticios y tasa de interés, que son variables financieras que tienen gran impacto en la economía de un país.

En la Tabla 3 se presenta un resumen de las crisis financieras de los últimos 27 años, periodo que abarca de 1991 al 2018, se muestra la relación que éstas guardan con los ciclos económicos y financieros de Estados Unidos y México.

Tabla 3. Crisis financieras de los últimos 27 años versus los Ciclos Económicos y Financieros de Estados Unidos y México.

\begin{tabular}{|c|c|c|c|c|c|c|c|c|c|}
\hline \multirow{2}{*}{\multicolumn{2}{|c|}{ Crisis }} & \multicolumn{4}{|c|}{ Ciclos Económicos } & \multicolumn{4}{|c|}{ Ciclos Financieros } \\
\hline & & \multicolumn{2}{|c|}{$\begin{array}{c}\text { Estados Unidos } \\
\text { (NBER) }\end{array}$} & \multicolumn{2}{|c|}{ México (SICCA) } & \multicolumn{2}{|c|}{ DJ } & \multicolumn{2}{|c|}{ IPC } \\
\hline Año & Nombre & Pico & Valle & Pico & Valle & Pico & Valle & Pico & Valle \\
\hline 1991 & Crisis Japonesa & & & mar-92 & oct-93 & & & nov-91 & nov-92 \\
\hline 1994 & Crisis Mexicana & & & jul-94 & oct-95 & & & oct-94 & abr-95 \\
\hline 1997 & Crisis Asiática y Rusa & & & & & & & nov-97 & oct-98 \\
\hline 2001 & Burbuja tecnológica & mar-01 & nov-01 & ago-00 & ago-03 & ene-00 & mar-03 & mar-00 & mar-03 \\
\hline 2007 & Hipotecas subprime & dic-07 & jun-09 & abr-08 & jun-09 & oct-07 & abr-09 & jun-07 & abr-09 \\
\hline 2010 & Deuda griega & & & & & & & ene-11 & oct-11 \\
\hline 2013 & Crisis ucraniana & & & & & & & ene-13 & feb-14 \\
\hline 2015 & Crisis del mercado financiero chino & & & & & mar-15 & ago-15 & abr-15 & dic-15 \\
\hline
\end{tabular}

Fuente: Elaboración propia con información de Claessens y Kose (2013), Mohieldin (2017).

La comparación entre ciclos económicos y financieros deriva de la evidencia de que los mercados financieros son influenciados por los ciclos económicos (Wong, 2016). Al detectar un ciclo financiero, se dice que se tiene un indicador del ciclo económico. 


\section{Metodología}

Para explicar la relación entre los ciclos financieros y los ciclos económicos de Estados Unidos y México se emplean conceptos y modelos ampliamente utilizados en el estudio de ciclos económicos. En esta sección se describe los pasos que sigue la metodología y conceptos que toma en cuenta, particularmente: tendencia, ciclo, puntos de giro y fechado de puntos de giro, conformidad y sincronía.

El primer paso, se realiza el procedimiento de descomposición de una serie de tiempo en tendencia y ciclo, haciendo uso del Filtro Hodrick y Prescott (HP) (1997). Posteriormente se aplica el algoritmo Bry y Boschan (B\&B) (1971), el cual está formulado conforme a la experiencia del NBER, para la determinación de puntos de giro y su fechado, con ello se logra la identificación de puntos máximos (picos) y mínimos (valles) locales de una serie en particular. Por último, se utilizan los conceptos de conformidad y sincronía para la calificación y clasificación de series de tiempo con base en su comportamiento, explicando así los co-movimientos de las series financieras y económicas, de acuerdo con Moore \& Shiskin (1967). Lo anterior revela una explicación que aún no es deducida en términos económico-financieros sobre la relación México-Estados Unidos, así como su relación con las crisis financieras más relevantes del periodo en estudio.

En las siguientes subsecciones se describe la metodología utilizada para definir un ciclo y para descomponer una serie de tiempo en tendencia y ciclo; posteriormente el algoritmo $\mathrm{B} \& \mathrm{~B}$ para fechar los puntos de giro en el ciclo de una serie de tiempo. Por último, se describen los conceptos de conformidad y sincronía.

\subsection{Ciclos económicos}

La definición de un ciclo de negocio por Burns y Mitchell (1946) y que es la comúnmente aceptada para Estados Unidos menciona lo siguiente:

Un ciclo consiste en expansiones que ocurren casi al mismo tiempo en diversas ramas de la actividad económica, seguidas en general por recesiones, contracciones y recuperaciones que se combinan en la fase de expansión del ciclo siguiente; esta secuencia de cambios es recurrente pero no periódica; en duraciones los ciclos de negocios varían de más de un año a diez o doce años. (Burns y Mitchell, 1946, p.3).

\subsection{Descomposición de una serie de tiempo en tendencia y ciclo}

De acuerdo con Guerrero (2011) para descomponer una serie de tiempo en términos de ciclos económicos, debe hacerse el supuesto de que una serie de tiempo se puede descomponer en dos componentes o variables no observables: la tendencia, que muestra un comportamiento suave, asociado con un patrón de largo plazo; y el ciclo, que se define como las fluctuaciones obtenidas a partir de la diferencia entre los datos observados y su respectiva tendencia (Hodrick y Prescott, 1997).

La estimación de la tendencia y el ciclo se puede realizar utilizando diversos métodos. Una forma no paramétrica de realizarlo es mediante Análisis Espectral (AE) de la serie y el correspondiente diseño y aplicación de Filtros pasa Banda (FPB). El AE de series temporales es útil para, entre otras cosas: ajuste estacional, procesamiento de imágenes, identificación de patrones; así también, es una herramienta útil para descubrir componentes periódicos, ya que consiste en expresar la serie de tiempo como una suma de sucesiones o componentes periódicos. Esta representación, por tanto, permite centrar la atención en frecuencias que más interesen; la tendencia y el ciclo de la serie por lo tanto están asociadas a determinados rangos de valores de frecuencias, o duración de los ciclos, que aparecen en el espectro de la serie y por lo tanto es posible extraer, resaltar o eliminar de la serie particular, mediante la aplicación de filtros pasa bandas diseñados para un fin específico.

Para la descomposición de la tendencia y ciclo existen diversos métodos, Canova (2007) 
señala que pueden clasificarse en tres tipos: estadísticos, económicos e híbridos (combinación de los dos anteriores). En este trabajo, se propone un procedimiento que combina el concepto de filtro pasa bandas y la aplicación de un método hibrido (estadísticoeconómico-financiero) para fijar tanto el porcentaje de suavidad de la tendencia como el rango de frecuencias del componente ciclo. El procedimiento estadístico sugerido es el uso del filtro de HP aplicado en forma de un filtro pasa bandas. La elección de los rangos de frecuencia o tamaño de los ciclos se seleccionaron con base en estudios empíricos y teorías acerca los ciclos económicos, tomando como referencia los rangos de frecuencia encontrados por Stock y Watson (1998).

El filtro de HP requiere de la elección de un parámetro de alisamiento, al cual se le asigna por lo común un valor estándar, en función de la periodicidad de observación de la serie. Es importante por consiguiente la elección adecuada de dicha constante que minimice distorsiones del filtro.

En particular el planteamiento base del filtro de HP es

$$
y_{t}=\tau_{t}+\eta_{t} \quad \text { para } t=1, \ldots N
$$

Donde $\left\{y_{t}\right\}$ es una serie de tiempo original, $\left\{\tau_{t}\right\}$ la tendencia estimada y $\left\{\eta_{t}\right\}$ el ciclo. La tendencia se estima al resolver el problema de minimización de la función,

$$
M\{\lambda\}=\sum_{t=1}^{N}\left(y_{t}-\tau_{t}\right)^{2}+\lambda \sum_{t=3}^{N}\left(\tau_{t}-2 \tau_{t-1}+\tau_{t-2}\right)^{2}
$$

Donde $\lambda>0$ es una constante que penaliza la variabilidad de la tendencia.

Referente a la selección de la constante de alisamiento $\lambda$, se sugiere elegirla en función del periodo de la actividad cíclica que se desea analizar, es decir definir el ciclo de referencia de $T$ meses, que divida la serie en dos componentes, el primero o tendencia, definido por los ciclos mayores a $\mathrm{T}$ meses (bajas frecuencias ${ }^{2}$ ), y el complemento con ciclos menores a $\mathrm{T}$ meses (altas frecuencias). La fórmula por utilizar es la propuesta por Maravall y del Río (2007):

$$
\lambda=\frac{1}{4 \times[1-\cos (2 \times \pi / T)]^{2}}
$$

Donde $\mathrm{T}$, es el tamaño del ciclo de referencia utilizado para dividir la serie en dos componentes.

Con esta fórmula, se puede aplicar el filtro HP a fin de producir el efecto de un filtro pasa bandas. La razón de usar este efecto es por la definición de su uso que menciona Guerrero:

El enfoque basado en filtros pasa bandas es desarrollado por la idea de que una definición matemática de lo que constituye un ciclo, puede conducir a un procedimiento que limpie apropiadamente la serie de fluctuaciones que no corresponden al movimiento periódico dentro de un cierto rango de duración de ciclos (Guerrero, 2011, p.53).

Como se mencionó anteriormente, se desea descomponer las series en dos componentes, tendencia y ciclo; éstos se pueden definir siguiendo lo propuesto por Stock y Watson (1998), quienes mediante estudios empíricos encontraron que el ciclo más corto (de cresta a cresta) es de seis trimestres (año y medio) y el más largo, de 39 trimestres (poco menos de diez años), asimismo, el $90 \%$ de los ciclos no duran más de 32 trimestres (ocho años). Por lo tanto, se puede definir los siguientes intervalos de duración de las fases: para la tendencia, mayores a diez años; y el componente ciclo eventos de duración mayores a año y medio y menores a diez años.

\footnotetext{
${ }^{2}$ La frecuencia $(w)$ se define con el reciproco del periodo $(\mathrm{T})$.
} 
La forma de obtener la tendencia de una serie y su componente ciclo con los rangos definidos anteriormente, es mediante la aplicación doble del filtro de HP: en la primera aplicación del filtro HP se utiliza el valor de $\lambda=133,107,938$ ( $\mathrm{T}=120$ meses, 10 años), el elemento tendencia que arroja este procedimiento será la tendencia de la serie, la cual incluye únicamente ciclos iguales o mayores a diez años. El residuo o ciclo, la diferencia entre la serie original y la tendencia, es una serie con ciclos menores a diez años, los cuales incluyen efectos de tipo estacional, "trading day", valores atípicos, así como patrones con ciclos menores a seis trimestres; la segunda aplicación del filtro de HP se realiza sobre el ciclo obtenido, para eliminar estos elementos y quedarse únicamente con patrones con ciclos de duración entre seis y 39 trimestres, el valor de la constante de alisamiento ahora se calcula con $\mathrm{T}=12$ meses, $\lambda=13,9$; el resultado que arroja esta segunda aplicación del filtro HP es una serie con fluctuaciones cíclicas que van de 12 a 120 meses, omitiendo tanto los efectos estacionales así como otros factores asociados a ciclos menores a 12 meses. En la Figura 4 se presenta un ejemplo de la serie original y su descomposición en tendencia y ciclo.

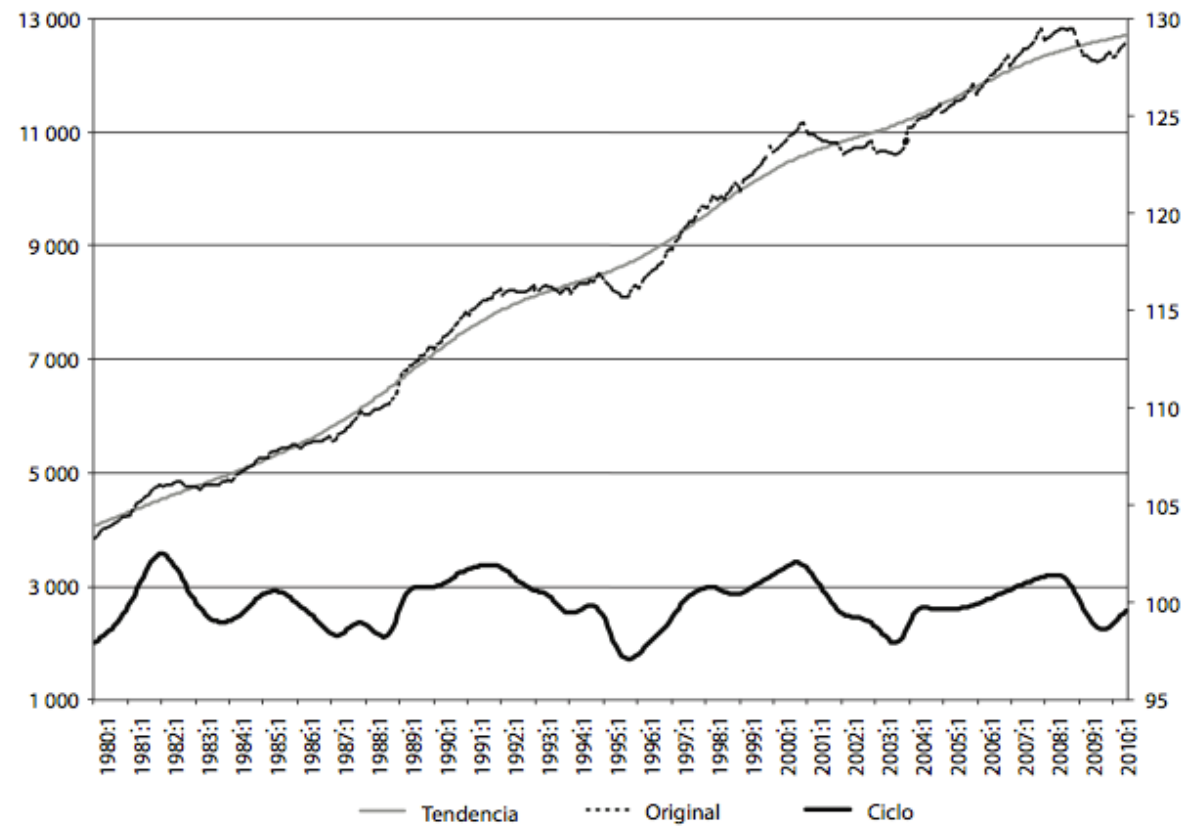

Figura 4. Series originales, de tendencia y ciclo, para las variables coincidentes Fuente: Serie del IMSS, Guerrero (2013).

\subsection{Identificación de puntos máximos (picos) y mínimos (valles), puntos de giro}

Para la identificación de puntos máximos (picos) y mínimos (valles) locales de una serie en particular la técnica de puntos de giro es una de las más utilizadas. En este sentido, el algoritmo B\&B (1971), es adecuado para la identificación de estos puntos en series de tiempo. Al obtener los puntos de giro, éstos se fechan y puede medirse el ciclo económico, ya sea de pico a pico o de valle a valle, (Antón, 2011). El algoritmo B\&B está formulado conforme a la tradición del NBER, que de acuerdo con Antón (2011) replica razonablemente bien los puntos de giro establecidos por el mismo NBER para la economía de Estados Unidos.

El algoritmo B\&B es utilizado por Mönch y Uhlig (2005), para el estudio de ciclos de negocio en series de tiempo europeas; así también, el INEGI lo utiliza para el fechado 
de los ciclos del SICCA. En particular la implementación del algoritmo utilizado aquí, es una adecuación al código de MatLab desarrollado por Mönch y Uhlig (2005).

Asimismo, Antón (2011) menciona que el algoritmo está diseñado de tal forma que la duración de un ciclo económico no sea menor a 15 meses y que las fases del ciclo (contracciones y expansiones) sean, al menos, de cinco meses. En la Figura 5 se muestra el fechado del indicador coincidente del SICCA, utilizando el algoritmo de B\&B.

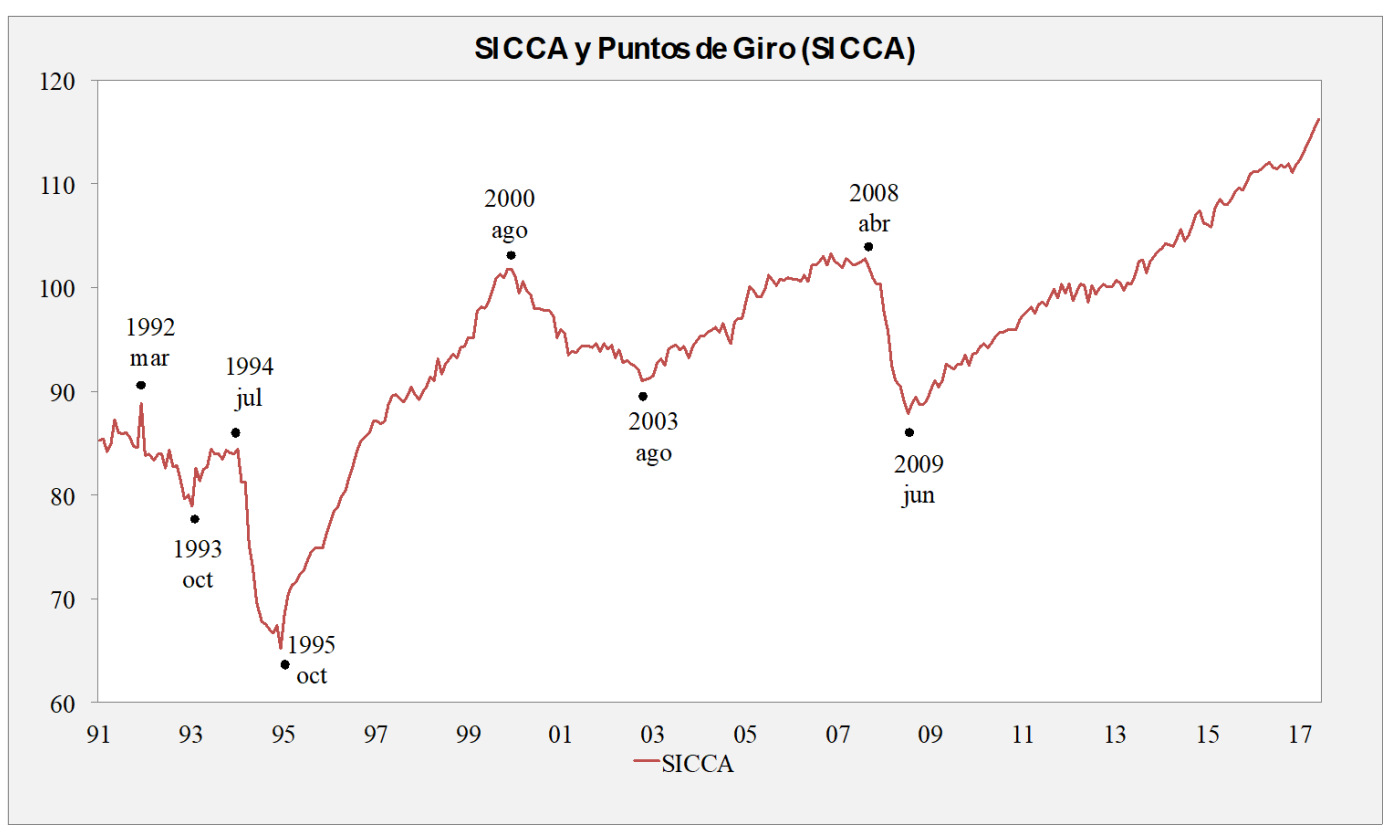

Figura 5. Índice Coincidente del SICCA de noviembre de 1991 a abril de 2018, con las fechas de sus puntos de giro determinadas con el algoritmo de B\&B.

Fuente: elaboración propia con información INEGI.

\subsection{Co-movimientos entre series de tiempo}

Uno de los temas fundamentales acerca el estudio de los ciclos económicos es la calificación y clasificación de series de tiempo con base en su comportamiento con respecto a un ciclo de referencia. Moore y Shiskin (1967) presentan un sistema cualitativo y cuantitativo de calificación y clasificación de indicadores cíclicos, para realizar este sistema utilizaron muchas de las ideas y modelos generadas por el grupo de académicos del NBER. Con este sistema se realiza el análisis de co-movimientos de las tendencias y ciclos previamente obtenidos de las series financieras y económicas, haciendo uso de dos conceptos propuestos por Moore y Shiskin, conformidad y sincronía, los cuales se describen a continuación.

\subsubsection{Conformidad}

De acuerdo con Moore \& Shiskin (1967), la conformidad se define como el comportamiento que una serie particular presenta con respecto a una de referencia; específicamente, la consistencia con la que los movimientos de una serie particular coinciden con los de la serie de referencia. La medida de la conformidad de una serie particular con respecto a una de referencia, indica si los movimientos de la serie tienen un movimiento paralelo consistente con los ciclos de referencia; esta medida verifica si una serie se expande consistentemente durante las expansiones de los ciclos de referencia y se contrae durante las recesiones. Una consideración importante es el hecho de que algunas series pueden contraerse durante las expansiones de la serie de referencia y expandirse durante las contracciones (series opuestas a los ciclos de negocio), o pueden mostrar características diferentes de sincronización 
en relación a los ciclos del negocio (se adelantan o atrasan). Es por esto, que es importante identificar los patrones de expansión y contracción de la serie particular y su relación con la serie de referencia, en cada ciclo de negocio. En la Figura 6 se muestra un ejemplo de conformidad entre las tendencias del componente coincidente del SICCA y la del DJ, se observa una alta conformidad al mostrar la consistencia con la que los movimientos a la alza y a la baja se realizan al mismo tiempo, así también su correlación es 0.96.

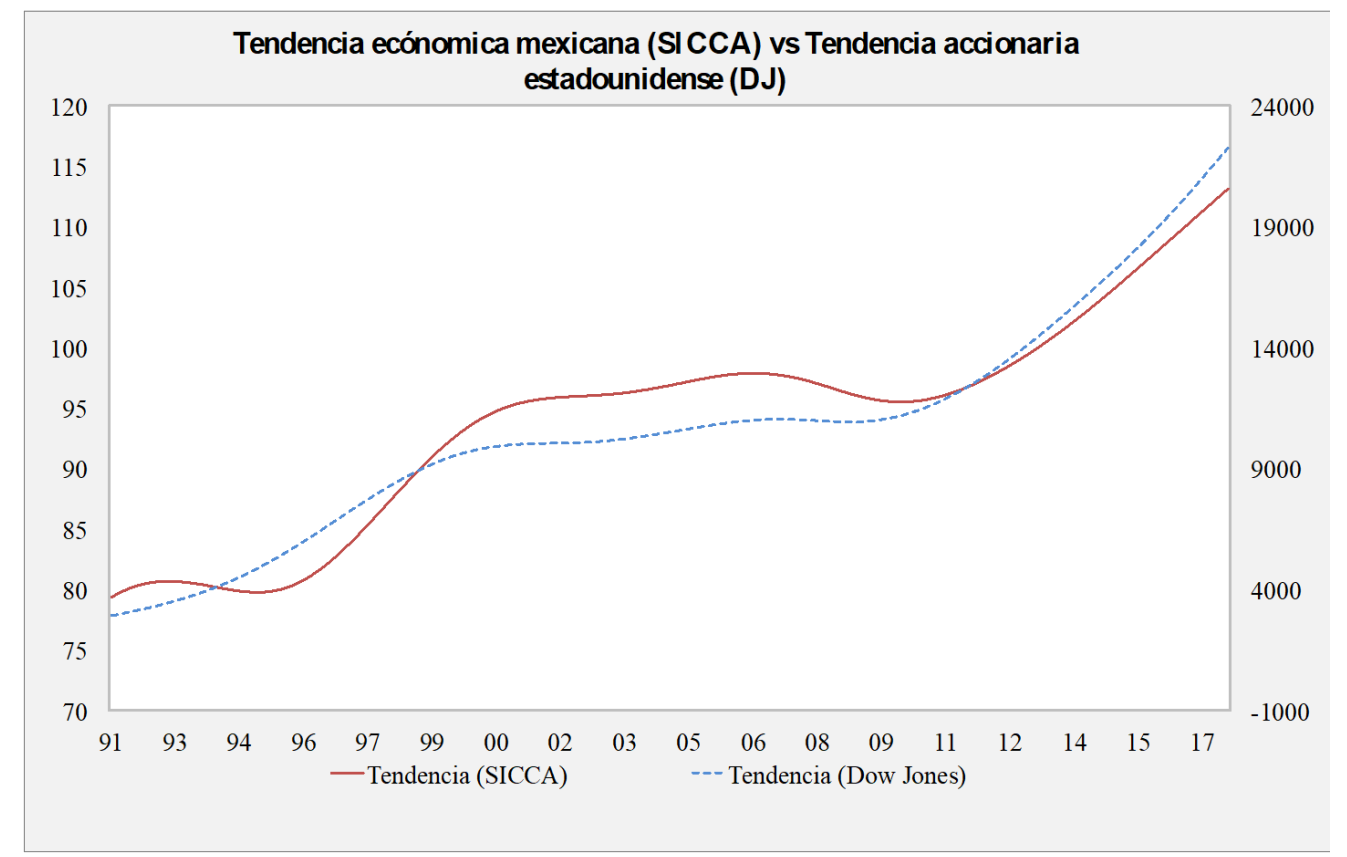

Figura 6. Tendencia del índice coincidente del SICCA versus tendencia del DJ de noviembre de 1991 a abril de 2018.

Fuente: elaboración propia con información de INEGI y Yahoo Finance.

\subsubsection{Sincronía}

Al igual que la conformidad, la sincronía está definida con base a una serie particular y una de referencia. Moore \& Shiskin (1967) consideran tres tipos de sincronía: adelantada, coincidente y rezagada. La sincronía entre los ciclos de la serie particular y la serie referencia se entiende como el comportamiento promedio en que las fechas de los picos y valles de la serie particular, análogos a los de la serie de referencia; coinciden, se adelantan o se atrasan. El primer paso para determinar la sincronía entre la serie particular con respecto a la de referencia, es emparejar los puntos de giro (picos o valles) de la serie particular con los de la serie de referencia, ciclos análogos.

En la Tabla 4 se muestra un ejemplo de sincronía entre los ciclos análogos del índice IPC respecto a los del DJ. En particular, se observa, que en dos ocasiones, los picos del IPC se rezagaron: dos meses en la crisis de la Burbuja tecnológica y un mes en el periodo de la crisis del mercado financiero chino, por lo que se podrían considerar casi coincidentes durante estas crisis, y solo en una ocasión se adelantó cuatro meses, Hipotecas subprime. Con respecto a los valles, en dos ocasiones las fechas del punto de giro del valle en ambas series fue coincidente y en una ocasión se atrasó cuatro meses. En conclusión, se puede decir que el IPC tiene coincidencia tanto en picos como en valles con el DJ. 
Tabla 4. Sincronización entre los ciclos análogos del IPC versus los del DJ.

\begin{tabular}{|c|c|c|c|c|c|c|c|}
\hline \multirow{2}{*}{\multicolumn{2}{|c|}{ Crisis }} & \multicolumn{4}{|c|}{ Ciclos Financieros } & \multicolumn{2}{|c|}{$\begin{array}{l}\text { Sincronía en meses } \\
\text { (IPC respecto a DJ) }\end{array}$} \\
\hline & & & & & & Pico & Valle \\
\hline Año & Nombre & Pico & Pico & Pico & Valle & Pico & Valle \\
\hline 2001 & Burbuja tecnológica & mar-00 & mar-03 & ene-00 & mar-03 & -2 (atrasada) & 0 (coincidente) \\
\hline 2007 & Hipotecas subprime & jun-07 & abr-09 & oct-07 & abr-09 & 4 (adelantada) & 0 (coincidente) \\
\hline 2015 & $\begin{array}{l}\text { Crisis del mercado } \\
\text { financiero chino }\end{array}$ & abr-15 & dic- 15 & mar-15 & agc & -1 (atrasada) & -4 (atrasada) \\
\hline
\end{tabular}

Fuente: Elaboración propia

\section{Resultados}

En esta sección se presentan los resultados y su análisis desde dos rangos de frecuencia de estudio: tendencia (frecuencias bajas) y ciclo (frecuencias altas). Primero se compara la tendencia (periodos mayores a diez años) del componente coincidente del SICCA con la tendencia de los dos índices accionarios (DJ e IPC). Posteriormente, se comparan los ciclos (periodos mayores a seis trimestres y menores a diez años) del componente coincidente del SICCA con los ciclos de los dos índices accionarios de Estados Unidos y México (DJ e IPC) así como con el fechado del NBER. Se da una explicación de los fenómenos observados asociándolos a crisis internacionales que impactaron en las estructuras económicas de ambos países.

\subsection{Análisis de tendencias}

En este apartado se analiza el componente tendencia en las series financieras versus las tendencias económicas. Se hace uso del Filtro HP, para descomponer las series del IPC, DJ y SICCA en: ciclo y tendencia. Así también, se emplean las fechas de puntos de giro de las series financieras, obtenidos con el algoritmo B\&B, para observar la relación entre crisis financieras en los índices bursátiles con los cambios estructurales de largo plazo en el indicador económico de México.

\subsection{1. Índice accionario DJ versus componente coincidente del SIC- CA versus puntos de giro del ciclo del DJ}

En la Figura 7 se muestra la tendencia económica mexicana obtenida a partir de la tendencia del componente coincidente del SICCA, la tendencia accionaria estadounidense a partir de la tendencia del DJ y se destaca en el fondo con cambios de barras los puntos de giro de los ciclos financieros del DJ.

En la Figura 7 se observa una alta conformidad entre la tendencia del índice accionario estadounidense DJ, con la tendencia del componente coincidente del SICCA, que representa a la economía mexicana. Se observa como a partir de la crisis del 2001 conocida como la "Burbuja tecnológica" ambas tendencias inician un proceso de desaceleración en crecimiento que termina hasta marzo de 2003, momento que coincide con un punto de giro del índice DJ, que da inicio a un nuevo movimiento alcista. Es claro como a partir del punto de giro del índice DJ en octubre de 2007 ambas series presentan una tendencia a la baja debido a la crisis subprime originada en Estados Unidos, que a la luz de las tendencias capturadas culmina en abril de 2009. Con base en lo anterior, es posible explicar la alta conformidad que ambas tendencias presentan, mientras una sube (fase de expansión), la otra también lo hace, de manera similar, cuando una tiende a la baja (fase de recesión), la otra le acompaña en su movimiento. Posterior a la recuperación del índice DJ en su punto de giro de abril 2009, la tendencia de éste y del SICCA han sido al alza (fase de expansión), a pesar de que se detectó un ligero cambio entre marzo y agosto de 2015 debido a la crisis del mercado financiero chino. 


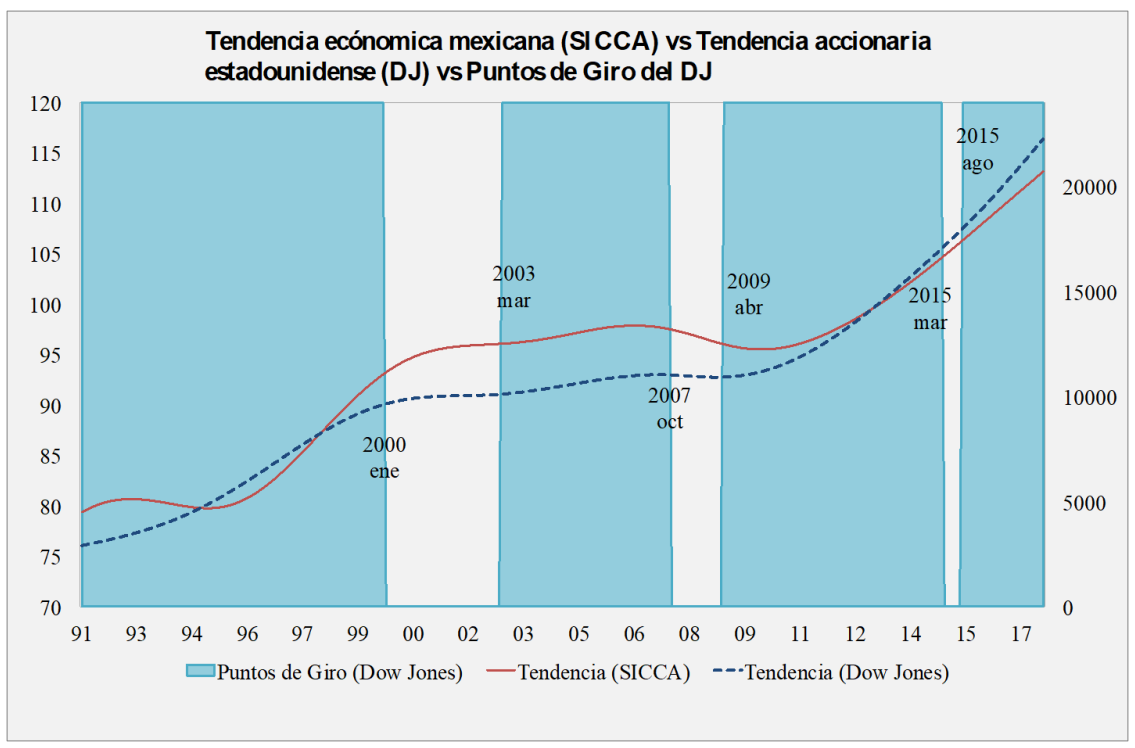

Figura 7. Comparación de la tendencia del indicador coincidente del SICCA versus la tendencia del DJ, las fechas señaladas son los puntos de giro de los ciclos del DJ.

Fuente: elaboración propia con información de INEGI y Yahoo Finance.

\subsection{2. Índice accionario IPC versus componente coincidente del SICCA versus puntos de giro del ciclo del IPC}

En la Figura 8 se muestra la tendencia económica mexicana obtenida a partir de la tendencia del componente coincidente del SICCA, la tendencia accionaria mexicana dada por la tendencia del IPC y se destaca en el fondo con cambios de barras los puntos de giro de los ciclos financieros del IPC.

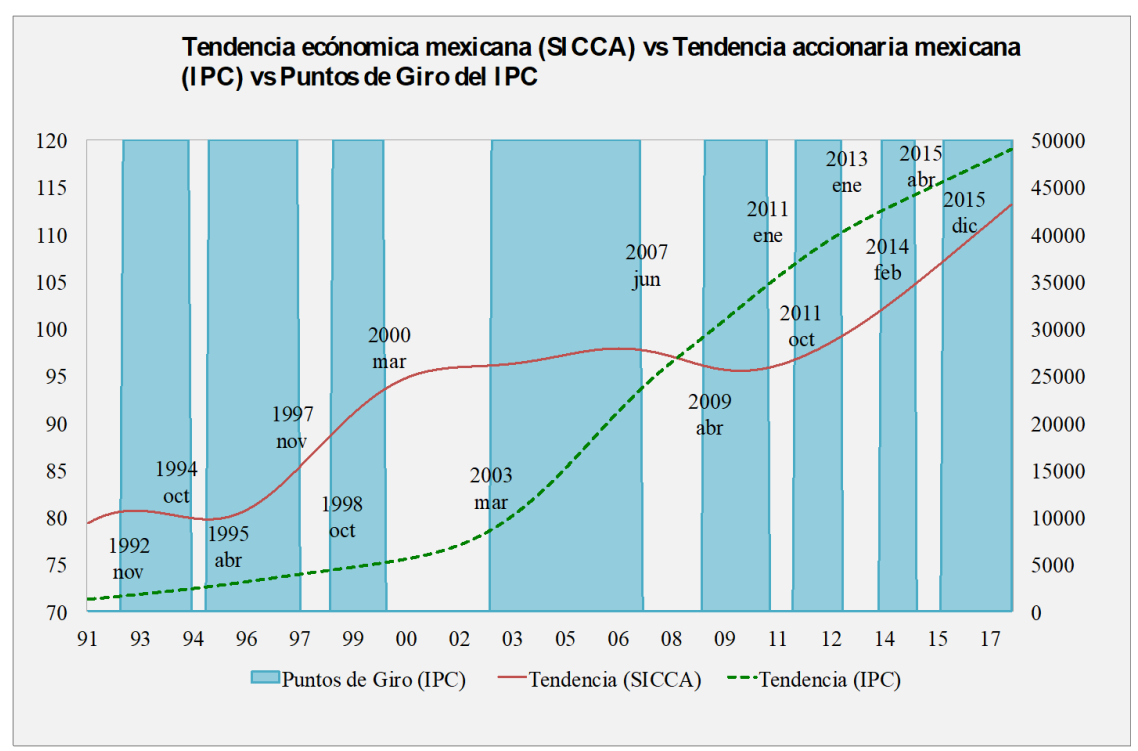

Figura 8. Comparación de la tendencia del indicador coincidente del SICCA versus la tendencia del IPC, las fechas señaladas son los puntos de giro de los ciclos del IPC.

Fuente: elaboración propia con información de INEGI y Yahoo Finance. 
En la Figura 8 se identifican tres momentos de especial interés que coinciden con eventos económicos relevantes; el primero de 1991 a 2001 donde se observa una ligera conformidad entre tendencias del SICCA e IPC, un segundo momento pero ahora de baja conformidad posterior al 2001, donde es notorio que la tendencia del índice accionario mexicano IPC es claramente a la alza, en tanto que la tendencia del SICCA es casi plana $\mathrm{y}$, finalmente, un tercer momento de elevada conformidad que comienza a partir de enero de 2011, es desde este momento que la conformidad alcista en tendencia se ha mantenido entre el SICCA e IPC.

Posterior a la Crisis Mexicana de 1994, en particular desde el punto de giro del IPC en abril de 1995, el comportamiento del SICCA muestra una tendencia a la expansión económica, siguiendo la tendencia del IPC. La crisis de 1994, a pesar de ser una de las más severas no afectó la tendencia del índice bursátil IPC. Es importante señalar que desde 1988 se había iniciado el proceso de liberalización financiera (Pacheco, 2005), lo cual representó perspectivas económicas favorables, seguida de los periodos de implementación de reformas estructurales y estabilización macroeconómica, acompañado de la entrada en vigor del TLCAN (1994), por lo que muchas empresas estadounidenses vieron oportunidades de crecimiento en México, y por supuesto la economía mexicana se vio beneficiada de las inversiones extranjeras productivas, lo cual se ve representado en la tendencia del SICCA a partir de 1996.

\subsection{Análisis de ciclos}

Para el análisis de ciclos, altas frecuencias, se hace uso del Filtro HP aplicado en dos ocasiones, con el objetivo de tener un filtrado pasa bandas para las series del IPC, DJ y SICCA, obteniendo así el componente ciclo, que se define como las fluctuaciones obtenidas de la diferencia entre los datos observados y su respectiva tendencia. Estas frecuencias consideran periodos mayores a seis trimestres y menores a diez años. También, se utilizan las fechas de los puntos de giro de las series financieras (IPC y DJ) y el SICCA; para el ciclo económico estadounidense se utiliza el fechado del NBER. Se muestra la sincronía en meses (coincidente, atrasada o adelantada) entre las series analizadas, con el objeto de explicar la relación entre los ciclos financieros y económicos con las crisis globales mencionadas para Estados Unidos y México.

\subsubsection{Análisis de los ciclos económicos: NBER (Estados Unidos) versus ciclo del componente coincidente del SICCA (México)}

En la Figura 9 se muestran los ciclos de la económica mexicana obtenidos a partir de los ciclos del componente coincidente del SICCA, y se destaca en el fondo con cambios de barras el fechado de los ciclos económicos del NBER para los Estados Unidos.

En la Figura 9 se observa una baja sincronía de la economía mexicana versus la economía estadounidense. Analizando la crisis del 2001 "Burbuja Tecnológica", ésta fue resentida en la economía mexicana siete meses antes que en la propia economía de Estados Unidos, sin embargo, la duración de la recesión en Estados Unidos duró apenas ocho meses, mientras que en la economía mexicana duró tres años. Por otro lado, durante la crisis subprime del 2007 el inicio de ésta fue cuatro meses antes en la economía estadounidense, diciembre de 2007, mientras que en México fue hasta abril de 2008, la recuperación de esta crisis fue coincidente en junio de 2009 para ambas economías. Lo anterior se complementa con lo mencionado por Antón (2011), quien señala que las recesiones para México tienen mayor duración que en países desarrollados, mientras que las expansiones son más cortas en México y más prolongadas en estos países. Se observa también como la Crisis Mexicana de 1994 no fue resentida por la economía estadounidense, ya que como se mencionó anteriormente fue un problema interno producto de contradicciones en la política fiscal y cambiaria en un entorno de globalización y liberalización financiera, además de incurrir en un riesgo moral por parte del sector bancario (Flores Paredes y Madrueño Aguilar, 
2006).

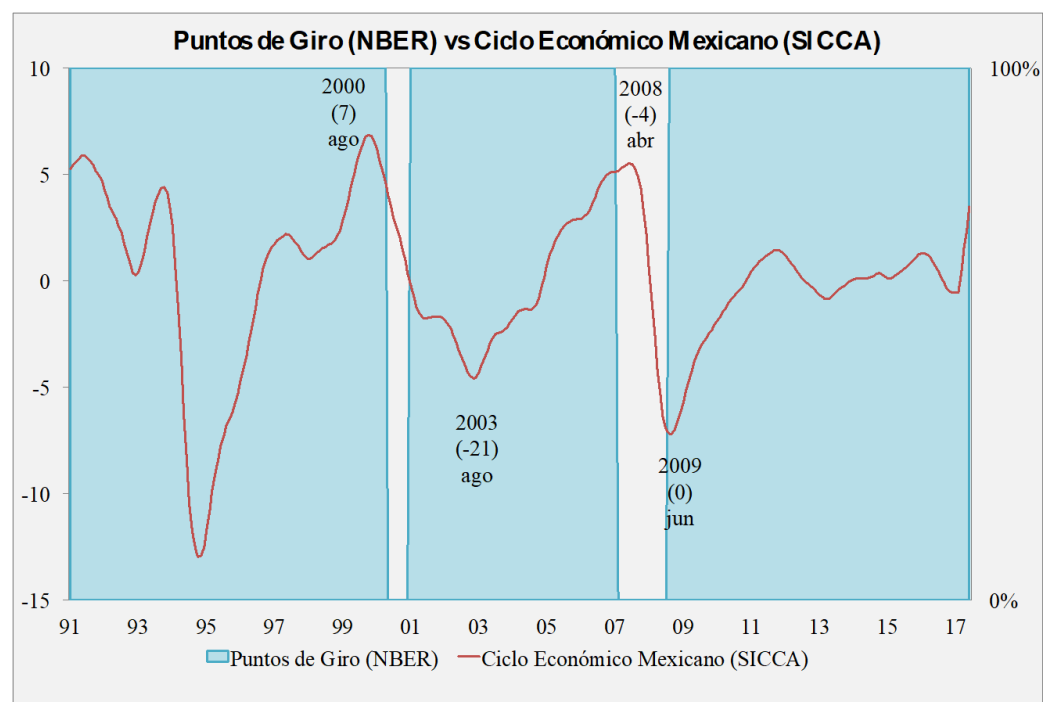

Figura 9. Fechado de los puntos de giro del NBER versus los ciclos del indicador coincidente del SICCA, las fechas señaladas son los puntos de giro del SICCA.

Fuente: elaboración propia con información de INEGI y NBER.

\subsubsection{Análisis de los ciclos financieros: de los índices accionarios DJ versus IPC}

En la Figura 10 se muestran los ciclos financieros mexicanos obtenidos a partir del índice del IPC, y se destaca en el fondo con cambios de barras los puntos de giro de los ciclos financieros del DJ para los Estados Unidos.

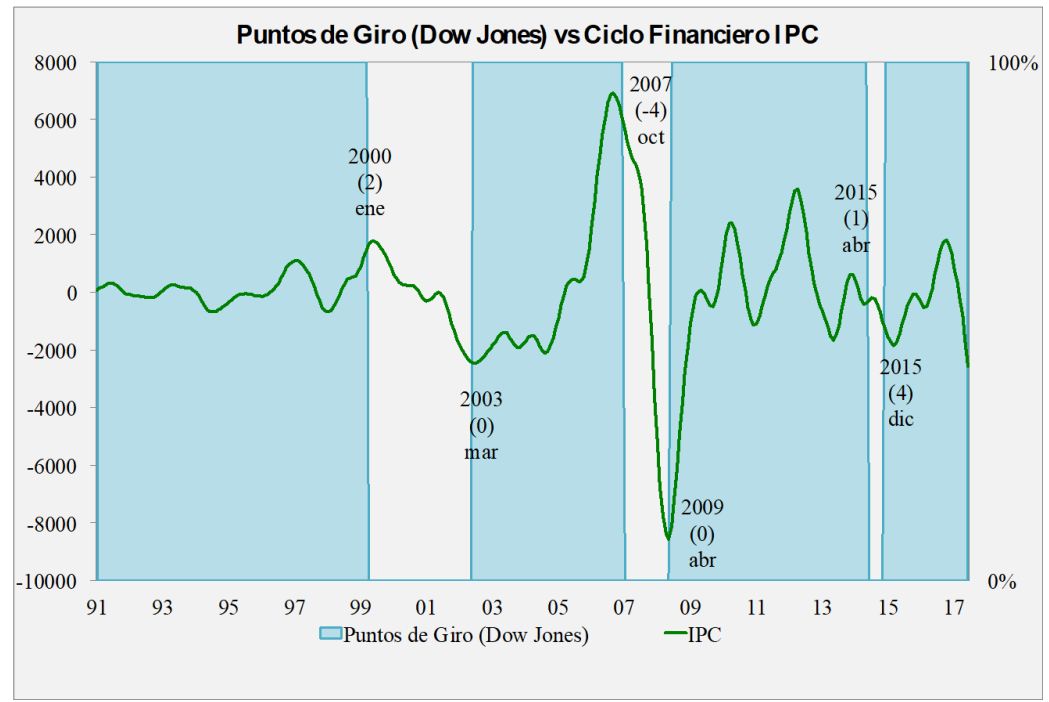

Figura 10. Fechado de los puntos de giro del DJ versus los ciclos del IPC, de noviembre de 1991 a abril de 2018, con las fechas de los puntos de giro del DJ determinadas con el algoritmo de B\&B.

Fuente: elaboración propia con información de Yahoo Finance.

En la Figura 10 se observa una sincronía casi coincidente de los ciclos análogos del DJ e IPC, ver Tabla 5, en promedio las fechas de los picos de ambas series coinciden y en 
los valles el DJ se adelanta un mes en promedio. Las dos crisis más relevantes de las que se ha hablado (2001-Burbuja tecnológica y 2007-subprime) fueron detectadas por ambos índices en fechas muy cercanas, prácticamente coincidentes. En la crisis de la burbuja tecnológica del 2001 el DJ adelantó por dos meses al IPC y su recuperación en ambos fue en marzo de 2003, en la crisis del 2007, el IPC adelantó con cuatro meses al DJ y su recuperación en ambos fue en abril del 2009. Son pocas las variaciones en que ambos ciclos no coinciden en expansiones y recesiones. Sin embargo, dado su comportamiento reciente podría pensarse que después de la crisis subprime del 2007 los ciclos de ambos índices accionarios tienen menos sincronía que en años anteriores, en particular la crisis griega del 2010 y la crisis ucraniana del 2013 no afectaron al DJ. Esto complementa lo mencionado por Díaz Rodríguez y Bucio (2017), analizando el contagio bursátil de mercados financieros, sugieren que México es un país vulnerable a los choques financieros de Estados Unidos y de otros países emergentes.

Tabla 5. Promedio de meses que adelanta el DJ al IPC.

\begin{tabular}{|c|c|c|c|c|c|c|c|}
\hline \multirow{2}{*}{\multicolumn{2}{|c|}{ Crisis }} & \multicolumn{4}{|c|}{$\begin{array}{l}\text { Ciclos Análogos } \\
\text { entre DJ e IPC }\end{array}$} & \multicolumn{2}{|c|}{$\begin{array}{l}\text { Sincronía en meses } \\
\text { (DJ respecto a IPC) }\end{array}$} \\
\hline & & \multicolumn{2}{|c|}{ IPC } & \multicolumn{2}{|r|}{ DJ } & Pico & Valle \\
\hline Año & Nombre & Pico & Valle & Pico & Valle & Pico & Valle \\
\hline 2001 & Burbuja tecnológica & mar-00 & mar-03 & ene-00 & mar-03 & 2 (adelantada) & 0 (coincidente) \\
\hline 2007 & Hipotecas subprime & jun-07 & abr-09 & oct-07 & abr-09 & -4 (atrasada) & 0 (coincidente) \\
\hline 2015 & $\begin{array}{l}\text { Crisis del mercado } \\
\text { financiero chino }\end{array}$ & abr-15 & dic-15 & mar- 15 & ago-15 & 1 (adelantada) & 4 (adelantada) \\
\hline & & & & & Promedio & 0 (coincidente) & 1(adelantada ) \\
\hline
\end{tabular}

Fuente: elaboración propia

\subsubsection{Análisis de los ciclos económicos y financieros: del índice ac- cionario DJ versus ciclo del componente coincidente del SICCA}

En la Figura 11 se muestran los ciclos económicos mexicanos obtenidos a partir del componente coincidente del SICCA, y se destaca en el fondo con cambios de barras los puntos de giro de los ciclos financieros del DJ para los Estados Unidos.

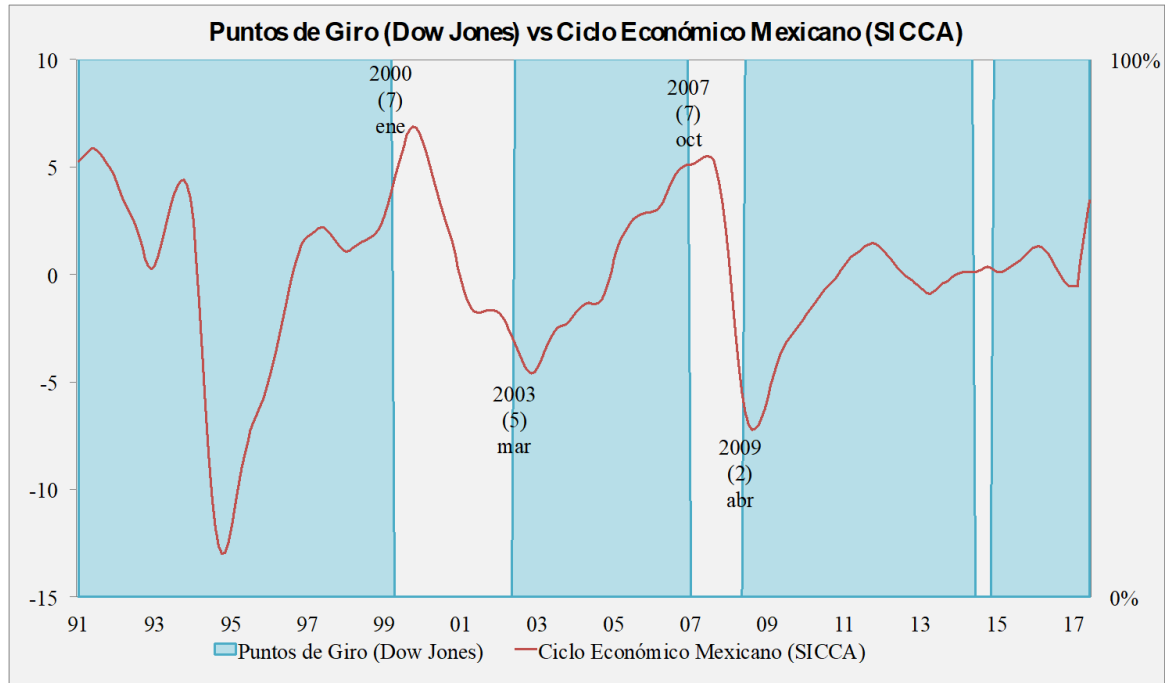

Figura 11. Ciclos del componente coincidente del SICCA versus Puntos de giro del DJ, de noviembre de 1991 a abril de 2018, con las fechas de los puntos de giro del DJ determinadas con el algoritmo de B\&B.

Fuente: elaboración propia con información de INEGI y Yahoo Finance.

En la Figura 11 es claro que en la dos crisis más importantes Burbuja Tecnológica 
(2001) y subprime (2007), el ciclo accionario estadounidense (DJ) adelantó al ciclo económico mexicano (SICCA) en el inicio de las recesiones (picos) en promedio siete meses, así también la fecha de recuperación en cuatro meses. Sin embargo, la crisis del mercado financiero chino (2015) no fue resentida por el ciclo de la economía mexicana, esto sugiere que existe una mayor relación en particular con el ciclo accionario estadounidense que con los ciclos financieros de otros mercados del mundo. Esto es de gran trascendencia pues quiere decir que los índices bursátiles compuestos por empresas estadounidenses de diversos sectores económicos resienten los efectos de las recesiones y expansiones mucho antes de que el efecto se presente en la economía real. Lo anterior tiene mayor sentido, si pensamos en que el mercado financiero a través de los precios ha descontado información y plasmado expectativas sobre eventos económicos trascendentes, ver Tabla 6.

Tabla 6. Promedio de meses que adelanta el DJ al SICCA.

\begin{tabular}{|c|c|c|c|c|c|c|c|}
\hline \multicolumn{2}{|c|}{} & \multicolumn{3}{c|}{$\begin{array}{c}\text { Ciclos Análogos entre DJ y SICCA } \\
\text { Cincronía en meses } \\
\text { Crisis }\end{array}$} & \multicolumn{2}{c|}{ DJ } & \multicolumn{2}{c|}{$\begin{array}{c}\text { Coincidente SICCA } \\
\text { Pico }\end{array}$} & Valle \\
\hline Año & Nombre & Pico & Valle & Pico & Valle & Pico & Valle \\
\hline 2001 & $\begin{array}{c}\text { Burbuja } \\
\text { tecnológica }\end{array}$ & ene-00 & mar-03 & ago-00 & ago-03 & 7 (adelantada) & 5 (adelantada) \\
\hline 2007 & $\begin{array}{c}\text { Hipotecas } \\
\text { subprime }\end{array}$ & oct-07 & abr-09 & abr-08 & jun-09 & 7 (adelantada) & 2 (adelantada) \\
\hline
\end{tabular}

Fuente: Elaboración propia

\subsubsection{Análisis de los ciclos económicos y financieros: del índice ac- cionario IPC versus ciclo del componente coincidente del SICCA}

En la Figura 12 se muestran los ciclos económicos mexicanos obtenidos a partir del componente coincidente del SICCA, y se destaca en el fondo con cambios de barras los puntos de giro de los ciclos financieros del IPC.

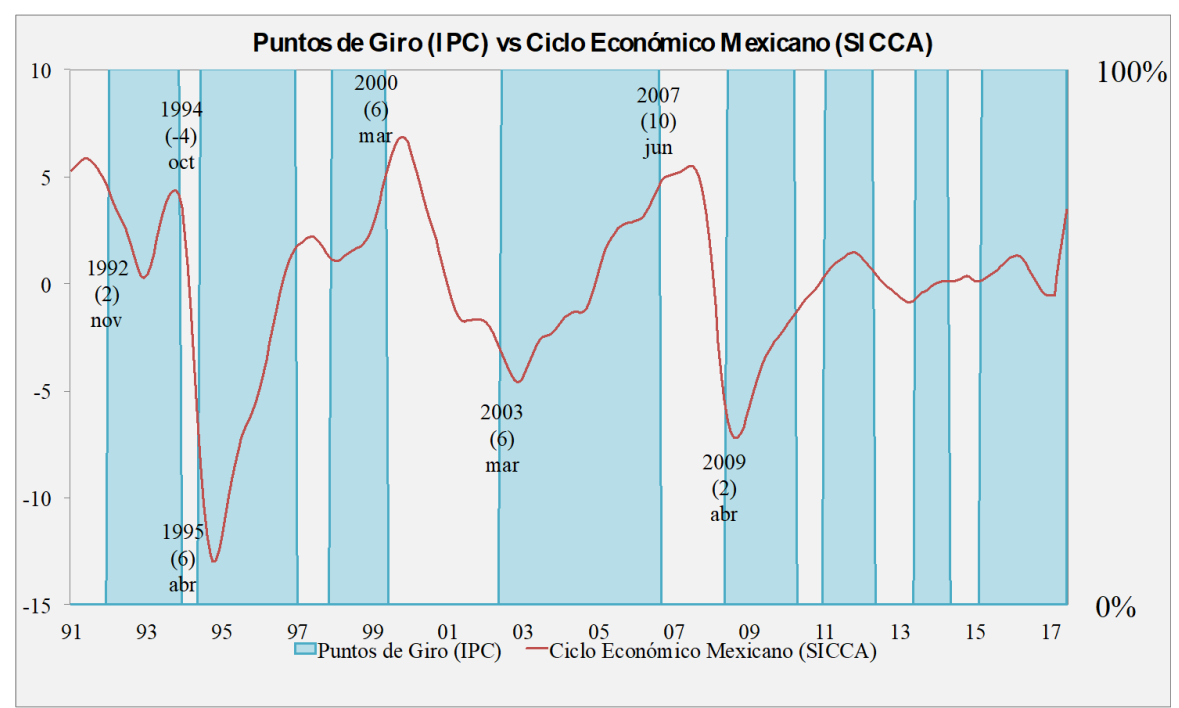

Figura 12. Ciclos del componente coincidente del SICCA versus Puntos de giro del IPC, de noviembre de 1991 a abril de 2018, con las fechas de los puntos de giro del IPC determinadas con el algoritmo de B\&B.

Fuente: elaboración propia con información de INEGI y Yahoo Finance. 
En la Figura 12 se observa que los ciclos del IPC muestra una sincronía adelantada con respecto al SICCA (economía mexicana). En particular, el IPC adelantó en promedio los picos cuatro meses y los valles seis meses a sus respectivos del SICCA. Como se mostró anteriormente, el índice accionario mexicano IPC reciente todas las crisis del periodo en estudio, no así la economía mexicana, la cual se ve afectada por crisis internacionales de gran envergadura como la Crisis Japonesa de 1991, que fue parte de la llamada "Década Pérdida", la crisis de la Burbuja Tecnológica del 2001 y la crisis subprime del 2007. Asimismo, el ciclo económico de México se ve afectado por crisis internas como la de 1994, que de acuerdo con Heath (2011) fue más severa que la del 2007, debido a que no se tuvo un control de la inflación y varios sectores productivos cayeron drásticamente. Una hipótesis parecida a la anterior es que las empresas mexicanas que conforman el IPC recienten mucho antes las recesiones y expansiones que el ciclo de la economía nacional, ver Tabla 7.

Tabla 7. Promedio de meses que adelanta el IPC al SICCA.

\begin{tabular}{|c|c|c|c|c|c|c|c|}
\hline \multirow{2}{*}{\multicolumn{2}{|c|}{ Crisis }} & \multicolumn{4}{|c|}{ Ciclos Análogos entre IPC y SICCA } & \multicolumn{2}{|c|}{$\begin{array}{c}\text { Sincronía en meses } \\
\text { (IPC respecto a SICCA) }\end{array}$} \\
\hline & & \multicolumn{2}{|c|}{ IPC } & \multicolumn{2}{|c|}{ Coincidente SICCA } & Pico & Valle \\
\hline Año & Nombre & Pico & Valle & Pico & Valle & Pico & Valle \\
\hline 1991 & Crisis Japonesa & nov-91 & nov-92 & mar-92 & oct-93 & 4(adelantada) & 11(adelantada) \\
\hline 1994 & Crisis Mexicana & oct-94 & abr-95 & jul-94 & oct-95 & $-4($ atrasada $)$ & 6(adelantada) \\
\hline 2001 & $\begin{array}{l}\text { Burbuja } \\
\text { tecnológica }\end{array}$ & mar-00 & mar-03 & ago-00 & ago-03 & $6($ adelantada $)$ & $6($ adelantada $)$ \\
\hline 2007 & $\begin{array}{l}\text { Hipotecas } \\
\text { subprime }\end{array}$ & jun-07 & abr-09 & abr-08 & jun-09 & 10(adelantada) & $2($ adelantada $)$ \\
\hline & & & & & Promedio & 4(adelantada) & 6(adelantada) \\
\hline
\end{tabular}

Fuente: Elaboración propia

\section{Conclusiones}

En este trabajo se obtuvieron dos conclusiones referente al análisis de las tendencias, la primera muestra la alta conformidad entre la tendencia del índice DJ y la tendencia del indicador coincidente del SICCA, comportamiento de largo plazo, éstas se expanden y contraen en los mismos periodos de tiempo. Esta actuación revela no solo la alta conformidad en términos de la series, sino también la elevada relación económico-financiera entre Estados Unidos y México, lo cual es un hecho ya conocido. Por otro lado, se observó que la tendencia del IPC presenta una sincronía mixta con respecto a la tendencia del indicador coincidente del SICCA, es decir, en ocasiones la tendencia accionaria mexicana de largo plazo no coincide con la dinámica económica nacional.

Respecto a la sincronía de los ciclos de las series accionarias versus los ciclos económicos, se encontró que en gran medida los ciclos financieros obtenidos con los índices accionarios (IPC y DJ) anticipan con al menos cuatro meses a los ciclos económicos de Estados Unidos y México, medidos a través del fechados del NBER y de los puntos de giro del SICCA, respectivamente. En cuanto al ciclo económico mexicano (SICCA) y el fechado del NBER para los ciclos de Estados Unidos presentan una baja sincronía.

En términos de crisis financieras, se concluye que durante la crisis interna en México (1994) los ciclos del IPC anticiparon cuatro meses en promedio las recesiones y recuperaciones de los ciclos económicos mexicanos, y no así el DJ. Es decir, el IPC sigue siendo un importante referente de la actividad económica interna de México. Por otro lado, cuando acontecen crisis financieras globales, por ejemplo, durante las dos grandes crisis originadas en los Estados Unidos (2001 y 2007) los ciclos del DJ adelantaron con un promedio de siete meses los picos y cuatro meses lo valles de la economía mexicana. En el mismo sentido el índice DJ anticipó las recuperaciones del fechado del NBER de Estados Unidos. Por lo tanto, el DJ es un buen indicador para adelantar cambios en los ciclos económicos.

Por último, la aportación metodológica de este trabajo es la de contribuir en un or- 
den lógico y práctico al análisis de series económicas y financieras retomando conceptos ampliamente reconocidos en el estudio de ciclos económicos, con el objetivo de explicar sus co-movimientos y su relación con las crisis financieras más importantes que han acontecido. Para futuras investigaciones, se recomienda complementar este trabajo con la incorporación de otras variables financieras como tasa de interés, ciclos crediticios, ciclos de vivienda y tasa de cambio, que escapan a este trabajo, para profundizar en la relación entre los ciclos financieros y económicos.

\section{Referencias}

Antón, A. (2011). El ciclo económico en México: características y perspectivas. Revista Internacional de Estadística y Geografía INEGI, 2 (2), pp. 32-49.

Beziz, P., Petit, G. (1997). The 1994 Mexican crisis: were signals inadequate?. Organisation for Economic Co-operation and Development (OECD), pp 1-10.

Bry, G., Boschan, C. (1971). Cyclical analysis of Time Series: Selected Procedures and Computer Programs. NBER Technical Report, pp. 7-63.

Burns, A.F., Mitchell, W.C. (1946). Measuring Business Cycles. National Bureau of Economic Research, pp. 1-22.

Calderón Villarreal, C., Hernández Bielma, L. (2017). Economic integration, economic crises and economic cycles in Mexico. Contaduría y Administración, 62, pp. 85-104.

Canova, F. (2007). Methods for Applied Macroeconomic Research. Princeton University Press. pp. 70-105.

Claessens, S., Kose, A. M. (2013). Financial Crises: Explanations, Types, and Implications. IMF Working Paper, 13 (28), pp. 1-66.

Claessens, S., Kose, A. M., Terrones, M. E. (2011). Financial Cycles: What? How? When?. IMF Working Paper, 11 (76), pp. 1-40.

Cruz Aké, S., Ramírez Alatriste, F., García Ruíz, R. S. (2012). Sincronización de fase en los mercados internacionales de capitales, evidencia de integración. Revista Mexicana de Economía y Finanzas, 7 (2), pp. 155-173.

Cuevas, A., Messmacher, M., Werner, A. M. (2003). Sincronización Macroeconómica entre México y sus Socios Comerciales del TLCAN . Dirección General de Investigación Económica Banco de México. pp 1-123.

Díaz Rodríguez H., Bucio, C. (2017). Contagio Bursátil en los mercados del TLCAN, países emergentes y el mercado global. Revista Mexicana de Economía y Finanzas, 13 (3), pp. 345-362.

Flores Paredes, J., Madrueño Aguilar, R. (2006). Ciclo económico, crisis financieras y choques externos, perspectivas de México. Comercio Exterior BANCOMEXT, 56 (8), pp. 642-657.

Grinderslev, O. J., Kramp, P. L., Krongborg, A. F., y Pedersen, J. (2017). Financial cycles: what are they and what do they look like in denmark?. Danmarks Nationalbank Working Papers, 115, pp. $1-53$.

Guerrero, V. M. (2013). Capacidad predictiva de los índices cíclicos compuestos para los puntos de giro de la economía mexicana. Economía Mexicana, 22 (1), pp. 47-99.

Guerrero, V. M. (2011). Medición de la tendencia y el ciclo una serie de tiempo económica, desde una perspectiva estadística. Revista Internacional de Estadística y Geografía INEGI, 2 (2), pp. 50-73.

Heath, J. (2011). Identificacion de los ciclos economicos en Mexico: 30 anos de evidencia. Revista Internacional de Estadistica y Geografía INEGI, 2 (2), pp. 19-31.

Hodrick, R. J., Prescott, E. C. (1997). Postwar U.S. Business Cycles: An Empirical Investigation. Journal of Money, Credit and Banking, 29 (1), pp.1-16.

INEGI (2010). Sistema de Indicadores Compuesto y Adelantado. Disponible en: http://internet. contenidos . inegi.org.mx/contenidos/Productos/prod_serv/contenidos/espanol/bvinegi/productos/derivada/ coyuntura/sicca/sicca.pdf

Jaramillo Olivares, A., Jaramillo Jaramillo, M. (2016). Crisis financiera del 2008: efecto en las empresas listadas en la Bolsa Mexicana de Valores. Revista Mexicana de Economía y Finanzas, 11 (3), pp. 67-83. DOI: https://doi.org/10.21919/remef.v11i3.26

Karfakis, C., Karfaki, E. (2018). Is the financial cycle a leading indicator of real output during expansions and contractions? A quantile analysis for Greece. The Journal of Economic Asymmetries, 18, pp. $1-10$.

Mönch, E., Uhlig, H. (2005). Towards a Monthly Business Cycle Chronology for the Euro Area. Journal of Business Cycle Measurement and Analysis, 2 (1), pp. 43-69.

Maravall, A., Del Río, A. (2007). Temporal Aggregation, Systematic Sampling, and the Hodrick-Prescott Filter. Computational Statistics 86 Data Analysis, 52 (2), pp. 975-998. 
REMEF (The Mexican Journal of Economics and Finance)

Mejía-Reyes, P., Campos-Chávez, J. (2008). Are the Mexican States and the United States Business Cycles Synchronized? Evidence from the Manufacturing Production . Economía Mexicana, 20 (1), pp. $79-112$.

Mohieldin, M. (2017). Ten Years After the Early Signs of the Financial Crisis: the State of the Economy and Economists. World Bank Group, Faculty of Economics and Political Science Cairo University.

Moore, G. H., Shiskin, J. (1967). Indicators of Business Expansions and Contractions. National Bureau of Economic Research, pp. 1-120.

NBER (2010).Recuperado de diciembre de 2018, Disponible en: https://m.nber .org/cycles/US_Business_ Cycle_Expansions_and_Contractions_20120423.pdf

Pacheco, P. (2005). Liberalización de la política comercial y crecimiento económico de México. Economía UNAM, 2 (4), pp. 84-93.

Rocha Armada, M. J., Leitão, J., Lobão, J. (2011). The contagion effects of financial crisis on stock markets: what can we learn from a cointegrated vector autoregressive approach for developed countries?. Revista Mexicana de Economía y Finanzas, 6 (1), pp. 29-53. DOI: https://doi.org/10. 21919/remef.v6i1.14

Samuelson, P., Nordhaus, W. (2002). Economía. McGrawHill. 17 edición, pp. 455-465.

Sosa, M., Ortiz, E., Cabello, A. (2017). Crisis financiera global y su impacto en la dinámica bursátil europea y americana. Revista Mexicana de Economía y Finanzas, 12 (3), pp. 1-27. DOI: https: //doi.org/10.21919/remef.v12i3.94

Stock, J. H., Watson, M. W. (1998). Business Cycle Fluctuations in U.S. Macroeconomic Time Series. NBER Working Papers Series, pp. 1-46.

Wong, T. (2016). Modeling Cyclical Markets-Part 1. Advisor Perspectives, pp. 1-11. 